\title{
Testing extra dimensions with boundaries using Newton's law modifications
}

\author{
V.K. Oikonomou ${ }^{1 *}$ and K. Kleidis ${ }^{2 \dagger}$ \\ ${ }^{1}$ Department of Informatics and Communications \\ Technological Education Institute of Serres \\ 62124 Serres, Greece \\ ${ }^{2}$ Department of Mechanical Engineering, Technological \\ Education Institute of Serres \\ 62124 Serres, Greece
}

December 8, 2018

\begin{abstract}
Extra dimensions with boundaries are often used in the literature, to provide phenomenological models that mimic the standard model. In this context, we explore possible modifications to Newton's law due to the existence of an extra-dimensional space, at the boundary of which the gravitational field obeys Dirichlet, Neumann or mixed boundary conditions. We focus on two types of extra space, namely, the disk and the interval. As we prove, in order to have a consistent Newton's law modification (i.e., of the Yukawa-type), some of the extra-dimensional spaces that have been used in the literature, must be ruled out.
\end{abstract}

\section{Introduction}

One of the most challenging perspectives in contemporary physics is the unified description of all the fundamental forces in nature within a self-consistent theoretical framework. In this context, a vast amount of theoretical work has been carried out during the last sixty years.

A successful effort to incorporate all interactions into a unified scheme is M-theory, together with its low energy limits, the Type IIA and Type IIB string theories, the $E_{8} \times E_{8}$ heterotic string theories [1-4], and so forth. These scenarios suggest that spacetime possesses additional compact spatial dimensions. Unfortunately, the corresponding compactification scale is much lower than the scales that can be experimentally examined. There are also string-inspired extensions of the four-dimensional quantum field theory, including large extra dimensions [5], in which the compactification scale is of the order of TeV. Such

*voiko@physics.auth.gr

${ }^{\dagger}$ kleidis@teiser.gr 
models appear to incorporate consistently the standard-model phenomenology [6] and, therefore, they could set a path towards higher-dimensional extensions of other quantumfield-theory areas (see [7 20], for an extensive though incomplete list). Some of these extensions could be verified, even experimentally [5]. In this context, it is expected that the existence of extra dimensions can be further scrutinized in the Large Hadron Collider, now operating at CERN. A potential experimental verification of the extra dimensions existence is quite challenging and, perhaps, the already developed experiments could be enriched also with others, corresponding to alternative types of approach [21 23].

A theoretical concept that could be verified experimentally in the near future, is the potential modification of gravity in three dimensions, due to the existence of extra dimensions. In fact, many theoretical studies have already been accounted for such a perspective 2429 . According to these models, the gravitational potential in three dimensions (Newton's law) can be modified, due to a compact $n$-dimensional extra space, thus resulting in Yukawa type corrections, which are of the form:

$$
V_{3}(r)=-G_{N} \frac{M M^{\prime}}{r}\left(1+a e^{-\frac{r}{m_{1}}}\right)
$$

where $r$ is the radial coordinate in three dimensions, $G_{N}$ is Newton's universal constant of gravitation in four spacetime dimensions, $a$ is the (dimensionless) strength of the gravitational field and $m_{1}$ is the length scale involved. According to Eq. (1), the correction to the Newtonian potential, $\frac{1}{r}$, is of the Yukawa type, $a e^{-\frac{r}{m_{1}}} / r$. In this context, $m_{1}=m^{-1}$, where $m$ is the mass of the lightest Kaluza-Klein state (which is the leading-order mode). At distances relatively-close to the length-scale of the extra dimensions, $R$, macroscopic modifications are expected, both to the strength and to the range of the gravitational force and the three-dimensional gravitational potential changes [24,26 29]. A possible modification of Newton's law, due to the existence of extra dimensions, could be revealed by several types of experiments [6]. As a consequence, there are also several bounds constraining the size and number of the extra dimensions [21,23, 28, 31 33. Nevertheless, as far as the modification of Newton's law is concerned, the strongest constraint comes from the Eötvos and Cavendish-type of experiments, which strongly restrict the parameters $a$ and $m_{1}$ [6]. Their results are consistent with the theoretical predictions of Newtonian gravity down to 200 microns [34] (2004 result), while more recent results suggest that Newtonian gravity is valid down to 56 microns [35].

In this letter, we explore possible Yukawa-type modifications to the gravitational potential in three dimensions, due to the existence of extra-dimensional spaces, at the boundary of which the extra dimensional component of the gravitational field obeys Dirichlet, Neumann or mixed boundary conditions. We consider two types of extra space, a disk with compact radius $R$ and the interval with length $2 \pi R$. The aforementioned spaces have been frequently used in the extra-dimensional phenomenology literature. The interval, in particular, has been used in [37] in order to break gauge symmetries. Various gaugesymmetry-breaking patterns arise using combinations of Dirichlet and Neumann boundary conditions (these boundary conditions are quite similar to orbifold boundary conditions). These theories can accommodate the standard model without introducing the Higgs field. Another perspective of the interval is studied in [36]. On the same footing, the Dirich- 
let disk and the Neumann disk have already been exploited [38, 39], offering interesting phenomenology to the extra-dimensional extensions of the standard model. These models describe a scalar theory built on a six-dimensional spacetime with a Dirichlet disk (of radius $R$ ) as the extra dimensional space. The radius $R$ serves as the intermediate scale. The Dirichlet disk is used in order that, the singularity involved, to be resolved. Such models also appear in string theory [38], where massless or very light degrees of freedom emerge upon turning on small couplings, in a framework which possesses only heavy degrees of freedom. Our findings constrain the set of the allowed boundary conditions that the above models use. Moreover we exclude some spaces because these provide unphysical Newton's law corrections. 1

This paper is organized as follows: In Section 2, we present the basic formulas that yield Yukawa-type corrections to Newton's gravitational potential in three dimensions, due to the existence of extra spatial dimensions. In this context, we explore possible modifications to Newton's law arising from a higher-dimensional spacetime with a disk as the extra space, at the boundary of which the gravitational field obeys either Dirichlet or Neumann boundary conditions. In Section 3, we analyze the interval involving all the potential combinations of boundary conditions. We generalize to the cases of two- and three-dimensional intervals and we compare our results with those corresponding to other well-studied extra-dimensional spaces (such as the 2 -sphere, the 3 -torus etc.). In addition, we perform a brief study of the mixed (Robin) boundary conditions. In Section 4, we check the consistency of our results with those of the experimental methods used to test the validity of Newton's law. Finally, we present our conclusions in Section 5.

\section{Gravitational potential and extra dimensions-the case of a disk}

Here, we recall the most important formulas related to the Yukawa-type corrections of the gravitational potential in three dimensions, due to the existence of extra spatial dimensions (for a detailed analysis see, e.g., [25]).

We consider a background of the form $M^{4} \times M^{n}$, where $M^{n}$ stands for a $n$-dimensional compact manifold (no topology is specified) and $M^{4}$ is the four-dimensional Minkowski spacetime used in contemporary particle physics. We assume that there exists a complete set of orthonormal harmonic functions, $\Psi_{m}(x)$, defined on $M^{n}$. These harmonic functions are eigenfunctions of the $n$-dimensional Laplace-Beltrami operator $\Delta_{n}$ defined on $M^{n}$, with eigenvalues $\mu_{m}^{2}$

$$
-\Delta_{n} \Psi_{m}=\mu_{m}^{2} \Psi_{m}
$$

Provided that the length-scale of the compact dimensions, as compared to the radial distance (from the source) of the three-dimensional space, is small, the Newton's law in

\footnotetext{
${ }^{1}$ Assuming that the extra dimensional component of the gravitational field, obeys the same boundary conditions as the rest of the fields in the theory.
} 
three dimensions results in (for a detailed analysis consult [25]),

$$
V_{3}(r)=-\frac{G_{N} M}{r} \sum_{m} \Psi_{m}^{*}(0) \Psi_{m}(0) e^{-\left|\mu_{m}\right| r} .
$$

As we have already mentioned, Eq. (3) is valid for large values of $r$, as compared to the internal dimensions radii, and, in fact, it can be expressed in the form

$$
V_{3}(r)=-\frac{G_{N} M}{r} \sum_{m} d_{m} e^{-\left|\mu_{m}\right| r},
$$

where $d_{m}$ denotes the degeneracy of the eigenvalue $\mu_{m}$. Eq. (4) is particularly useful to those cases where the representative eigenfunction is too difficult to be found. This is due to the fact that the eigenvalues $\mu_{m}$ depend on the irreducible representation of the gravitational field and not only on the particular representative $\Psi$ [25].

\subsection{The Dirichlet disk as an extra-dimensional space}

Consider that the extra-dimensional space is a Dirichlet disk of radius $R$. Upon consideration of the aforementioned analysis, the overall gravitational potential is written in the form

$$
V_{3+2}=\sum_{m} \Phi_{m}(r) \Psi_{m}(x),
$$

where $\Psi_{m}$ represents the extra-dimensional counterpart of the gravitational potential, corresponding to harmonic functions on a two-dimensional disk, at the boundary of which they obey Dirichlet boundary conditions, i.e., $\Psi_{m}(R, \phi)=0$. The disk is parameterized by the radius $r$, with $0 \leq r \leq R$ and by the angle $\phi$, with $0 \leq \phi \leq 2 \pi$. The solutions to the Laplace equation (2) reads,

$$
\Psi_{m}(r, \phi)=J_{m}\left(\frac{x_{m n}}{R} r\right) e^{i m \phi}
$$

In this case, according to the solution of the eigenvalue equation (2), the gravitational potential in three dimensions reads

$$
V_{3}(r)=-\frac{G_{N} M}{r} \sum_{m, n} J_{m}^{*}(0) J_{m}(0) e^{-\left|x_{m n}\right| \frac{r}{R}},
$$

where $J_{m}$ are the Bessel functions of the first kind and $x_{m n}$ are their roots 2 In view of the particular special functions properties, the only non-zero term of Eq. (7) is the one containing $J_{0}(0)=1$, since, for $n \neq 0, J_{n}(0)=0$. Therefore, Eq. (7) results in

$$
V_{3}(r) \simeq-\frac{G_{N} M}{r} \sum_{n} e^{-\left|x_{0 n}\right| \frac{r}{R}} .
$$

\footnotetext{
${ }^{2}$ Note that, in Eq. (3), the value $x=0$ corresponds to $r=0$ and $\phi=0$, using the disk parametrization.
} 
The first root of $J_{0}$ is $x_{01}=2.40483$ [40, hence, in this case, the gravitational potential reads

$$
V_{3}(r) \simeq-\frac{G_{N} M}{r} e^{-2.40483 \frac{r}{R}}
$$

We kept only the leading order corrections to Newton's law (the next to leading order terms are exponentially suppressed, and therefore are subdominant terms). Eq. (9) implies a rather unexpected result, in the sense that a compact space with radius in the submillimeter scale should yield the four-dimensional Newton's law plus a small correction of the Yukawa-type form. Hence, although it is interesting, it appears that the Dirichlet disk cannot serve as an extra dimensional space, since it results in an unexpected form of Newton's law, namely that of the Yukawa type. Consequently, the extra-dimensional theories with the Dirichlet disk as extra space must be ruled out.

\subsection{An internal disk with Neumann boundary}

Now, we explore the modifications to Newton's gravitational potential in three dimensions in the case where the extra two-dimensional disk obeys Neumann boundary conditions. The Neumann disk was used in [38], along the same lines as those in the Dirichlet case described in the previous section. In the Neumann case, the harmonic function $\Psi(x)$ obeys $\left.\frac{\mathrm{d} \Psi}{\mathrm{d} x}\right|_{x=R}=0$ and the gravitational potential in three dimensions is written in the form

$$
V_{3}(r)=-\frac{G_{N} M}{r} \sum_{m, n} J_{m}^{*}(0) J_{m}(0) e^{-\left|x_{m n}^{\prime}\right| \frac{r}{R}},
$$

where $x_{m n}^{\prime}$ are the roots of the Bessel function's derivative $J_{m}^{\prime}(x)$. The only non-zero term involved in the sum on the rhs of Eq. (10) is the one containing $J_{0}(0)=1$ and, therefore, $V_{3}(r)$ reads

$$
V_{3}(r) \simeq-\frac{G_{N} M}{r} \sum_{n} e^{-\left|x_{0 n}^{\prime}\right| \frac{r}{R}}
$$

The first root of the derivative of the Bessel function involved is $x_{01}^{\prime}=0$, while, the corresponding second root (which is the lowest Kaluza-Klein mode) is $x_{02}^{\prime}=3.8317$. Subsequently, keeping the lowest Laplacian eigenvalues $x_{01}$ and $x_{02}$, Eq. (11) results in

$$
V_{3}(r) \simeq-\frac{G_{N} M}{r}\left(1+e^{-3.8317 \frac{r}{R}}\right) .
$$

In the above equation we kept only the leading order corrections to Newton's law (which don't include subdominant terms). We observe that, in this case, due to the properties of the harmonic functions attributed to the internal disk (with Neumann boundary), the three-dimensional gravitational potential acquires the usual form of Newton's law, $\frac{1}{r}$, plus a Yukawa-type correction, reminiscent to compact extra dimensions. Thereupon, a twodimensional internal space compactified on a disk with Neumann boundary, could result in the Yukawa-type modification of Newton's gravitational potential, in contrast to the corresponding Dirichlet case. 


\section{The Interval}

In this section we study another extra-dimensional space, that has been used in several multi-dimensional extensions of the standard model [36,37. It is conceptually very simple, corresponding to an one-dimensional interval of length $2 \pi R$, denoted as $I=[0,2 \pi R]$. The spacetime structure is of the form $M^{4} \times I$, with $M^{4}$ being the four-dimensional Minkowski spacetime. In this article, we are not interested in the wealth of phenomenological features that this specific model may offer [36, 37]. Instead, we explore the way that the threedimensional gravity is modified. The literature distinguishes four cases with respect to the boundary conditions admitted at $x=0$ and at $x=2 \pi R$, namely, Dirichlet-Dirichlet (D-D), Dirichlet-Neumann (D-N), Neumann-Dirichlet (N-D), and Neumann-Neumann (NN) 37. It is easy to see that the gravitational corrections of the D-N and N-D extra spaces are unphysical, since there is no zero mode corresponding to these boundary conditions. Indeed the eigenvalues of the Laplace-Beltrami operator are $n+1 / 2$, with $n=0,1,2,3, \ldots$. Hence, we have a similar situation to the Dirichlet disk we studied earlier. On the other hand, the D-D case also yields unphysical results, since

$$
V_{3}(r) \sim \sum_{n} \sin (0)^{2} e^{-\lambda_{n} r / R}
$$

which is zero (with $\lambda_{n}$ the D-D eigenvalues). The only case that predicts Yukawa-type corrections is the N-N case. Accordingly, the harmonic function, $\Psi(x)$, of the gravitational potential along the extra spatial dimension $(x)$ obeys

$$
\left.\frac{\mathrm{d} \Psi}{\mathrm{d} x}\right|_{x=2 \pi R}=0,\left.\quad \frac{\mathrm{d} \Psi}{\mathrm{d} x}\right|_{x=0}=0
$$

and the corresponding eigenfunctions are of the form

$$
\Psi(x) \simeq \cos \left(\frac{n x}{2 R}\right), \quad n=0,1,2, \ldots
$$

The correction to the three-dimensional gravitational potential is given by,

$$
V_{3}(r)=-\frac{G_{N} M}{r} \sum_{n} d_{n} e^{-n \frac{r}{2 R}}
$$

with $d_{n}$ being the degeneracy of the eigenvalues of the Laplace-Beltrami operator along the extra dimension. Once again, keeping the leading-order corrections to Newton's law (omitting subdominant terms), we obtain

$$
V_{3}(r)=-\frac{G_{N} M}{r}\left(1+e^{-\frac{r}{2 R}}\right) .
$$

The generalization of the interval to two and three dimensions 3 (the lengths of the intervals are considered equal) is straightforward and the potential equals to:

$$
\begin{aligned}
& V_{3}(r)=-\frac{G_{N} M}{r}\left(1+2 e^{-\frac{r}{2 R}}\right)(\text { Two Dimensional Interval }) \\
& V_{3}(r)=-\frac{G_{N} M}{r}\left(1+3 e^{-\frac{r}{2 R}}\right)(\text { Three Dimensional Interval })
\end{aligned}
$$

\footnotetext{
${ }^{3}$ The two dimensional space is of the form $M^{4} \times I \times I$, while the three dimensional is $M^{4} \times I \times I \times I$.
} 
It is assumed that the extra dimensional component of the field, obeys Neumann boundary conditions at all boundaries.

Summarizing, an extra space with Neumann boundaries could yield Yukawa-type corrections to Newton's law, in contrast to the extra spaces with Dirichlet, or combinations of Dirichlet and Neumann boundary conditions. This result is quite important, since it rules out a large number of extra-dimensional spaces.

\subsection{Interval with Robin Boundaries}

Now, we consider that, at the first boundary of the interval, $x=0$, the extra-dimensional component of the gravitational field, $\Psi$, obeys Robin boundary conditions 43 ] of the form,

$$
\left(1+\beta_{1} \partial_{x}\right) \Psi(x)=0
$$

and, similarly, on the second boundary, $x=2 \pi R$,

$$
\left(1+\beta_{2} \partial_{x}\right) \phi(x)=0,
$$

where $\beta_{1,2}$ are arbitrary constants. Robin boundary conditions are known as providing conformal invariance for field theories [43], and have been used in phenomenological models such as those of [37].

We shall give a general rule that must be obeyed in order to obtain the expected Yukawa type behavior of the gravitational corrections. Therefore we must address the spectral problem, subject to the Robin boundaries. The roots (which we denote $y_{n}$ ) of the equation,

$$
\left(1-b_{1} b_{2} y_{n}^{2}\right) \sin y_{n}-\left(b_{1}+b_{2}\right) y_{n} \cos y_{n}=0
$$

where $b_{i}=\beta_{i} / 2 \pi R$, are the eigenvalues of the extra space's Laplace-Beltrami operator, with the gravitational field obeying Robin boundary conditions [43]. If we denote the corresponding eigenfunctions $\varphi_{n}(x)$, then in order to have the Yukawa type corrections (and to avoid unphysical results) the eigenvalues must have a zero mode and the eigenfunctions must be non zero at $x=0$, namely:

$$
\varphi_{n}(0) \neq 0 \text {. }
$$

Then, the corrections of the gravitational potential take the form,

$$
V(r)=-\frac{G_{N} M}{r}\left(1+\sum_{n \neq 0} \varphi(0)^{*} \varphi(0) e^{-y_{n} r}\right)
$$

which is the physically accepted (and formally expected) behavior stemming from a compact extra space. It is obvious that the cases D-D, N-D, D-N, and N-N are subcases of the Robin boundary conditions. 

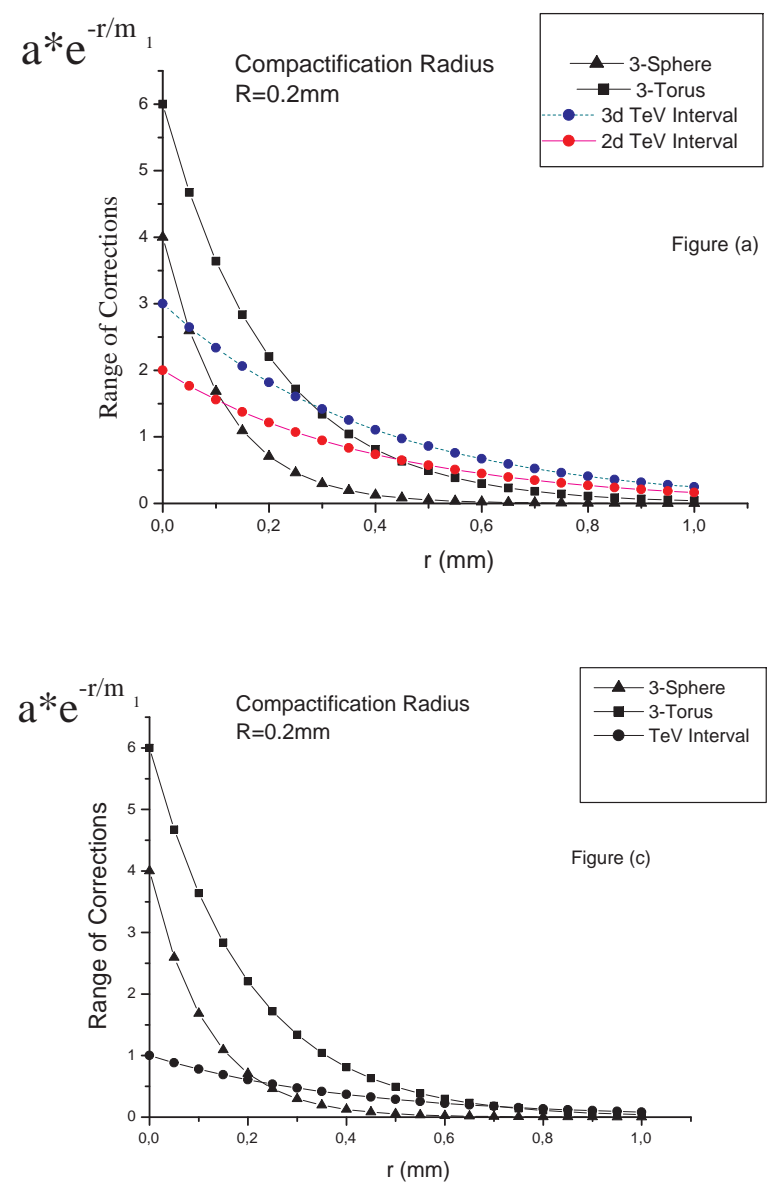

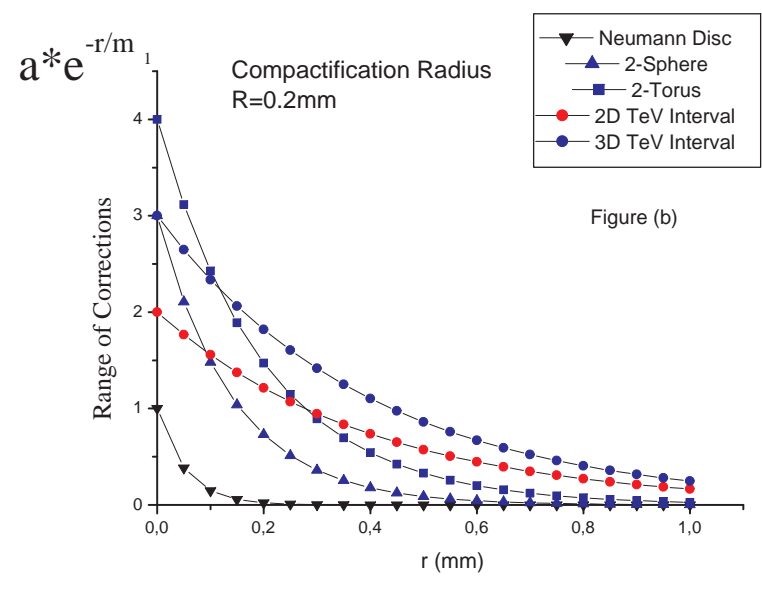

Figure 1: Comparison of the 1d, 2d, 3d interval with Neumann boundaries, with 2-Torus, 3-Torus, 2-Sphere, 3-Sphere and the Neumann disk. The compactification radius is equal to $R=0.2 \mathrm{~mm}$. The 2-dimensional spaces are shown in Figure (b) and the 3dimensional spaces are shown in Figure (a). The shadowing effects are clearly shown in Figures (a), (b) and (c). In Figure (c) the shadowing effect occurs even for spaces with different spatial dimensions, namely two 3dimensional with a 1-dimensional one.

\section{Analysis and Comparison of the Results}

In this section we check upon the consistency of our results with the experimental ones on the validity of Newton's law. First of all, we compare the behavior of the gravitational potential along the physically-acceptable spaces we found (the Neumann disk and the Neumann interval) to those admitted by a 2-dimensional torus, $T^{2}$, and a 2-dimensional sphere, $S^{2}$, as well as their three-dimensional counterparts, the 3 -sphere and the 3 -torus. Assuming that the torus radii are equal, and keeping the lowest Kaluza-Klein mode (i.e., to leading-order correction of the Newtonian potential), the gravitational potential corresponding to the n-torus is given by [25],

$$
V_{3}(r)=-\frac{G_{N} M}{r}\left(1+2 n e^{-\frac{r}{R}}\right),
$$

while, as far as the n-sphere is concerned, the result is quite similar [25],

$$
V_{3}(r)=-\frac{G_{N} M}{r}\left(1+(n+1) e^{-\frac{\sqrt{n} r}{R}}\right) .
$$


In Figs. 1 and 2, we plot the Yukawa-type correction-term, $a e^{-r / m_{1}}$, versus $r$, corresponding to the $1 \mathrm{~d}, 2 \mathrm{~d}, 3 \mathrm{~d}$ interval with Neumann boundaries, as well as to the 2-Torus, 3 -Torus, 2-Sphere, 3-Sphere and the Neumann disk. In Fig. 1 the compactification radius is equal to $R=0.2 \mathrm{~mm}$, while in Fig. 2 the compactification radius is equal to $R=0.05 \mathrm{~mm}$. These two values are quite near to the 2004 and 2006 experimental bounds, that is $0.2 \mathrm{~mm}$ and $0.056 \mathrm{~mm}$, respectively.

From Fig. 1 it becomes evident that, the corrections to the gravitational potential due to the 2-Torus and the 2-Sphere are larger, than those of the Neumann disk. This result also holds if the compactification radius is smaller, for example $R=0.05 \mathrm{~mm}$, see Fig. 2 (b). Observing Fig. 1 (a), which is the plot of the Yukawa corrections corresponding
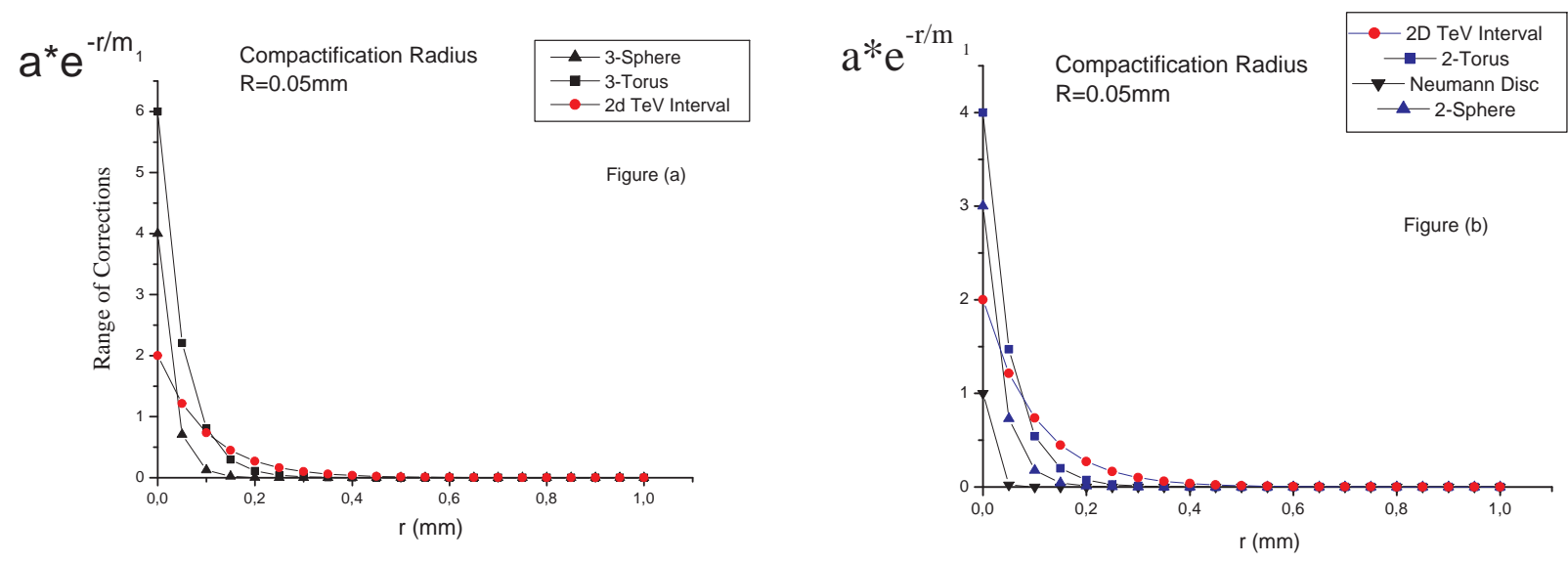

Figure 2: Comparison of the 1d, 2d, 3d inter-

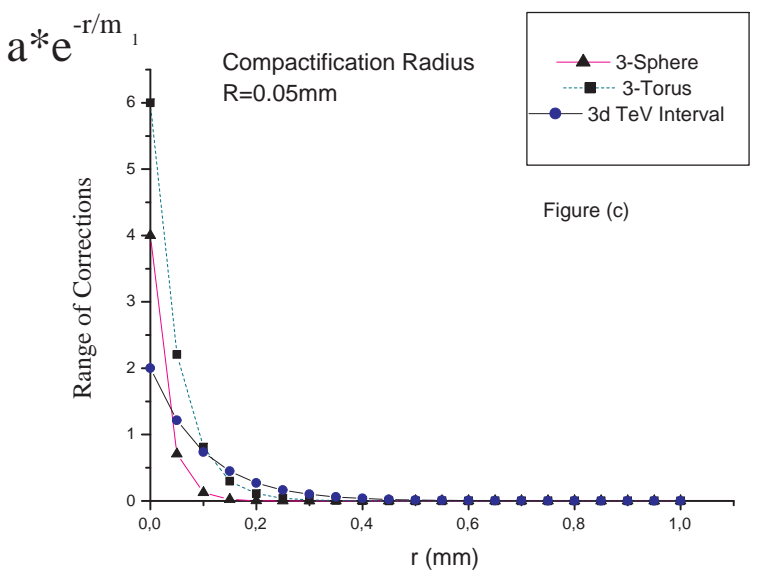
val with Neumann boundaries, with 2-Torus, 3-Torus, 2-Sphere, 3-Sphere and the Neumann disk. The compactification radius is equal to $R=0.05 \mathrm{~mm}$. The corrections are much smaller compared to the $R=0.2 \mathrm{~mm}$ case. In Figs. (a) and (c) we compare the 3sphere and the 3-torus with the two- and the three-dimensional interval, where the shadowing effect between spaces with different dimensions is clearly shown. In Fig. (b) we compare all the 2-dimensional spaces. We can see that the Neumann disk yields negligible corrections when compared to the other spaces.

to the 3-Sphere, 3 -Torus, the $2 \mathrm{~d}$ and $3 \mathrm{~d}$ interval, we can see that the $\mathrm{N}-\mathrm{N} 2 \mathrm{~d}$ and the corresponding 3d interval's corrections can become smaller, equal (at some point) and larger than the corrections corresponding to the torus- and the sphere-case. This also holds 


\begin{tabular}{|c|c|}
\hline Extra Dimensional Space & Radius, $\mathbf{R}$ \\
\hline 3-Torus $T^{3}$ & $\mathrm{R}=0.056 \mathrm{~mm}$ \\
\hline 2-Torus $T^{2}$ & $\mathrm{R}=0.056 \mathrm{~mm}$ \\
\hline 2-Sphere $S^{2}$ & $\mathrm{R}=0.079 \mathrm{~mm}$ \\
\hline 3-Sphere $S^{3}$ & $\mathrm{R}=0.096 \mathrm{~mm}$ \\
\hline Neumann disk & $\mathrm{R}=0.2145 \mathrm{~mm}$ \\
\hline 1-Dimensional Interval & $\mathrm{R}=0.028 \mathrm{~mm}$ \\
\hline 2-Dimensional Interval & $\mathrm{R}=0.028 \mathrm{~mm}$ \\
\hline 3-Dimensional Interval & $\mathrm{R}=0.028 \mathrm{~mm}$ \\
\hline
\end{tabular}

Table 1: The Radii of the compact spaces consistent with the experimental constraints on $m_{1}$

for the 1d interval (see Fig. 1 (c)). Such a behavior could arise problems, since we cannot figure out the space that corresponds to the measured corrections, reflecting an interesting phenomenon (often appearing in extra-dimensional theories) known as shadowing [41,42]. Similar results are obtained when $R=0.05 \mathrm{~mm}$, but the corrections are quite smaller than those corresponding to the $R=0.2 \mathrm{~mm}$ case. We must note that the plots appearing in Fig. 1 are for illustrative purposes only, since the value of the compactification radius, namely $R=0.2 \mathrm{~mm}$, is only compatible to the 2004 experimental results, but excluded by the 2006 experiment. In addition the plots for the interval in Fig. 2 are also for illustrative purposes, since the maximum allowed value for the radius corresponding to the interval space is smaller than $R=0.05 \mathrm{~mm}$ (see the list below and Table 1 ).

In view of Figs. 1 and 2, we need to stress that, if the compactification radius is small, the corrections near the current experimental limit are negligible. Nevertheless, we expect that, if the experiments allow us to check Newton's law at smaller distances, the corrections might be significant, as it is indicated also by [34] and 35.

Before closing this section, we must address another important issue that has to do with the experimental verification of the spaces we described above. The experiments verify Newton's law down to $0.056 \mathrm{~mm}$, with $95 \%$ confidence level [34,35. In this case, the experimental constraints are quite restrictive since [35]:

- For one extra dimension, the radius $R$ must be smaller than $0.044 \mathrm{~mm}$, that is $R \leq 44 \mu \mathrm{m}$

- For two equal-size extra dimensions, the common radius $R$ must be smaller than $0.0374 \mathrm{~mm}$, that is $R \leq 37 \mu \mathrm{m}$

- For $a \geq 1$, all radii of compactification with $m_{1} \geq 0.056 \mathrm{~mm}$ are excluded.

We can estimate the size of each compact space we studied, in order that the theoretical results to be consistent with the experimental constraint $m_{1} \geq 0.056 \mathrm{~mm}$. In Table 1 we present the predictions for the radii of each compact space. 
The only results which are not consistent with the experimental constraints are the 2 -sphere and the Neumann disk. Therefore both these extra spaces must be excluded.

\section{Conclusions}

In this article we studied possible modifications of Newton's law, due to the existence of a compact extra-dimensional space, at the boundary of which the extra-dimensional component of the gravitational field obeys Dirichlet, Neumann or combination of these boundary conditions. Usually, such a modification implements Yukawa-type corrections, $\frac{a}{r} e^{-r / m_{1}}$, in addition to the $\frac{1}{r}$ gravitational potential. We considered two types of extra spaces, namely the disk of radius $R$ (two-dimensional) and the compact interval, $I=$ $[0,2 \pi R]$ in one, two and three dimensions. In the Dirichlet-disk case, the results obtained are rather unexpected, i.e., the gravitational potential in three dimensions takes the form of a Yukawa-type potential and not of a Yukawa-type correction. Clearly, such a behavior is quite far from physical reality, since the well-established Newton's law, $\frac{1}{r}$, cannot be obtained. This result suggests that extra-dimensional models employing Dirichlet disks as extra spaces must be ruled out. On the contrary, an internal disk with Neumann boundaries does predict Yukawa-type corrections to the Newtonian potential, although, quantitatively, they are less significant than those arising from other two-dimensional spaces (cf. Figs. 1 and 2). However, this type of extra space must also be excluded, since the corresponding corrections to Newton's law do not compromise the recent experimental results (the same is also true for the 2 -sphere).

There is another extra-dimensional spacetime which yields corrections to the threedimensional gravitational potential, in which the extra space corresponds to the interval $I=[0,2 \pi R]$. In connection to the one-dimensional interval, we also studied the corresponding two- and three-dimensional intervals. We examined all the possible boundary conditions that were used in the extra-dimensional phenomenological models, using the interval as an extra space. We proved that the only acceptable combination (in reference to a correct behavior of the Newton's law corrections) is the interval with Neumann boundaries, i.e., our results rule out the Dirichlet boundaries and combinations between Dirichlet and Neumann boundaries.

Finally, let us note that the interval as an extra dimensional space, is the simplest structure that an extra space can have. Indeed, using the interval as an extra dimensional space, we come across only to the problem of explaining how the extra dimensions became so small and stabilized. When one considers for example the torus, an additional problem must be dealt with, which is the question why the extra space topology is non-trivial, but the other visible three have trivial topology. This makes the interval quite appealing as an extra space.

Acknowledgements: Financial support by the Research Committee of the Technological Education Institute of Serres, under grant SAT/ME/260111-01/02, is gratefully

\footnotetext{
${ }^{4}$ Nevertheless, we must note, that even the three visible dimensions of our world may have non-trivial topology. This exotic scenario is based on the assumption that the Universe topology can be different from Euclidean beyond the present day particle horizon [44.
} 
acknowledged.

\section{References}

[1] Polchinski J 1998, String Theory, Cambridge Monographs on Mathematical Physics

[2] Leontaris G K 2008, IJMP A23, 2055

[3] Leontaris G K 2009, IJMP A24, 6035

[4] Johnson C V 2003, D-Branes, Cambridge Monographs on Mathematical Physics

[5] Antoniadis I, Arkani-Hamed N, Dimopoulos S and Dvali G R 1998, Phys. Lett. B436, 257

[6] Kribs G D 2006, Tasi 2004 Lectures on the Phenomenology of Extra Dimensions, arXiv: hep-ph/0605325

[7] Buchbinder I L and Odintsov S D 1988, IJMP A3, 1859

[8] Buchbinder I L and Odintsov S D 1989, IJMP A4, 4337

[9] Odintsov S D 1988, Mod. Phys. Lett. A3, 1391

[10] Lavrov P M, Odintsov S D and Tyutin I V 1988, Mod. Phys. Lett. A3, 1273

[11] Kirsten K 1991, J. Phys. A24, 3281

[12] Kirsten K 1992, J. Phys. A25, 6297

[13] Kirsten K 1993, J. Phys. A26, 2421

[14] Elizalde E 2008, J. Phys. A41, 304040

[15] Elizalde E, Santos F C and Tort A C 2003, IJMP A18, 1761

[16] Elizalde E and Tort A C 2004, Mod. Phys. Lett. A19, 111

[17] Oikonomou V K 2009a, Rev. Math. Phys. 21, 615

[18] Oikonomou V K 2009b, arXiv: 0905.1465 [hep-ph]

[19] Oikonomou V K and Tracas N D 2010c, IJMPA 255935

[20] Oikonomou V K 2010a, Mod. Phys. Lett. A25, 767

[21] Kazanas D, Nicolaidis A 2003, Gen. Rel. Grav. 35, 1117

[22] Domokos G, Erdas A and Kovesi-Domokos S 2003, Astropart. Phys. 20, 215

[23] Oikonomou V K, Vergados J D and Moustakidis C C 2007, Nucl. Phys. B773, 19 
[24] Floratos E G and Leontaris G K 1999, Phys. Lett. B465, 95

[25] Kehagias A and Sfetsos K 2000, Phys. Lett. B472, 39

[26] Kehagias A and Russo J G 2000, JHEP 0007, 027

[27] Oikonomou V K 2008, Class. Quantum Grav. 25, 195020

[28] Floratos E G, Leontaris G K and Vlachos N D 2010, arXiv:1008.0765 [hep-ph]

[29] Oikonomou V K 2010b, arXiv: 1009.5222 [hep-ph]

[30] Randall L and Sundrum R 1999, Phys. Rev. Lett. 83, 3370

[31] Olive K A 2003, TASI Lectures on Dark Matter, arXiv: 0301505 [astro-ph]

[32] Vergados J D, Moustakidis C C and Oikonomou V K 2006, AIP Conf. Proc. 878, 138

[33] Hooper D 2009, TASI 2008 Lectures on Dark Matter, arXiv: 0901.4090 [hep-ph]

[34] Hoyle C D, Kapner D J, Heckel B R, Adelberger E G, Gundlach J H, Schmidt U and Swanson H E 2004, Phys. Rev. D70, 042004

[35] Hoyle C D, Kapner D J, Heckel B R, Adelberger E G, Gundlach J H, Schmidt U and Swanson H E, Phys. Rev. Lett. 98, 021101 (2007)

[36] Diego D and Quiros M 2008, Nucl. Phys. B805, 148

[37] C. Csaki, C. Grojean, H. Murayama, L. Pilo, J. Terning, Phys. Rev. D69, 055006 (2004)

[38] Dudas E and Papineau C 2006, JHEP 0611, 010

[39] Dudas E, Papineau C and Rubakov V A 2006, JHEP 0603, 085

[40] Abramowitz M and Stegun I 1970, Handbook of mathematical functions, Dover, New York

[41] Dienes K R, Mafi A 2002, Phys. Rev. Lett. 88, 111602

[42] Oikonomou V K 2011, Commun. Theor. Phys. 55101

[43] A. Romeo, A. A. Saharian, J. Phys. A35, 1297 (2002)

[44] De Oliveira-Costa A, Smoot G F and Starobinsky A A 1996, Astrophys. J. 468457 